\title{
Cytoplasmic Signaling Involved in Sonoporation- Induced Apoptosis and Mitosis Repression of Myeloid Leukemia Cells
}

\author{
Wenjing Zhong*, Wai-Hung Sit ${ }^{\dagger}$, Jennifer M. F. Wan ${ }^{\dagger}$, and Alfred C. H. Yu ${ }^{*}$ \\ * Medical Engineering Program ${ }^{\dagger}$ School of Biological Sciences \\ The University of Hong Kong, Pokfulam, Hong Kong SAR \\ Corresponding email: alfred.yu@hku.hk
}

\begin{abstract}
Sonoporation is known to be a transient phenomenon that may disrupt the homeostasis of living cells. In this work, we showed that sonoporation may bear time-lapse impact on cellular viability through up-regulation of cytoplasmic signaling proteins related to apoptosis and cell-cycle arrest. Our experiments were done on HL-60 leukemia cells $\left(10^{6}\right.$ cells $\left./ \mathrm{ml}\right)$, and sonoporation was induced via the use of $1 \% \mathrm{v} / \mathrm{v}$ microbubbles and $1-\mathrm{min}$. pulsed ultrasound exposure (0.5MPa peak negative pressure, $1 \mathrm{MHz}$ center frequency, $10 \%$ duty cycle, $1 \mathrm{kHz}$ pulse repetition frequency). The transient nature of sonoporation in these cells was confirmed by performing scanning electron microscopy on selected cell samples that were fixed respectively after a few seconds into the ultrasound exposure and one minute after the end of exposure. Cytoplasmic signaling changes of these cells were studied at four post-sonoporation time points $(4 \mathrm{~h}, 8 \mathrm{~h}, 12 \mathrm{~h}$, 24h) using western blot analysis. Five signaling proteins related to apoptosis and mitosis were analyzed in this work: 1) PARP (for DNA repair); 2) cleaved-PARP (fragments due to cleavage by pro-apoptotic caspase proteins); 3) Bcl-2 (inhibitor for mitochrondrial release of pro-apoptotic molecules); 4) Bax (complement of Bcl-2); 5) Cdc-2 (regulator for cell mitosis). Three key results were found from the cytoplasmic signaling analysis. First, PARP levels were reduced over the monitoring period whilst cleaved-PARP had increased in expression, and in turn they indicate that the cells' anti-apoptotic responses were dampened following sonoporation and pro-apoptotic caspase proteins were likely activated. Second, drop in Bcl-2 and rise in Bax were observed, and these suggest that the mitochondrion was involved in apoptotic signal transduction inside sonoporated cells. Third, Cdc-2 was seen to decrease, implying that mitosis was repressed in sonoporated cells.
\end{abstract}

Keywords—sonoporation, cytoplasmmic signals, apoptosis, mitosis.

\section{INTRODUCTION}

When acoustic cavitation develops in the vicinity of living cells, its mechanical interactions (between ultrasound and microbubble gas cavities) may exert a force that is substantial enough to create pores on the cell membrane [1]. This event is commonly known as sonoporation, and it is generally accepted as a transient phenomenon in which the membrane pores can reseal within an order of seconds [2]. In biomedicine, sonoporation has been considered as a potential strategy for facilitating cellular uptake of external molecules such as drugs and genes [3]. Since ultrasound can be focused deep into tissues to trigger sonoporation non-invasively, this drug delivery paradigm may represent a suitable alternative over electroporation-based approaches that often require insertion of electrodes to treatment sites away from the skin surface (e.g. the liver) [4].

Given that sonoporation involves physical alteration of the cell membrane structure, it may well disrupt the homeostasis of living cells and thereby upset their viability [5]. Indeed, it has been shown that sonoporation would affect the cells' resting membrane potential [6] and increase free radical formation [7]. These may represent confounding factors in using sonoporation for drug delivery purposes. To better understand the side effects of sonoporation, it is essential to examine the postsonoporation cell viability against different dosage parameters. Previous studies have shown that cells are generally viable shortly after experiencing sonoporation if imaging-range ultrasound parameters and microbubble densities are used [8]. However, concerns remain over whether sonoporation would affect the cell fate in the long run by triggering apoptosis events (i.e. programmed cell death) [9], [10]. If the long-term survival of sonoporated cells is limited, then cellular repair strategies like calcium ion chelation may be needed for sonoporation-based drug delivery paradigms [11].

In this work, we have examined how sonoporation brings about time-lapse effects on the cell fate by investigating the cytoplasmic signaling activities of sonoporated cells via the western blot technique. Our focus has been on studying the signaling pathways related to apoptosis and cell division up to 24 hours after sonoporation. The findings reported in this work are based upon in vitro studies conducted on HL-60 leukemia cells that are naturally deficient in p53 pro-apoptosis proteins.

\section{EXPERIMENTAL METHODS}

\section{A. Cell Culturing Procedure}

The acute promyelocytic leukemia HL-60 cell line was used in our study, and it was obtained from American Type Culture Collection (ATCC; Cat. No. CCL-240). The cells were cultivated as single-cell suspensions at $37^{\circ} \mathrm{C}$ in a $5 \% \mathrm{CO}_{2}$ atmosphere, and they were maintained using fresh Iscove's Modified Dulbecco's Medium (IMDM; ATCC, 30-2005) and 10\% Fetal Bovine Serum (FBS; ATCC, 30-2020). Cells 


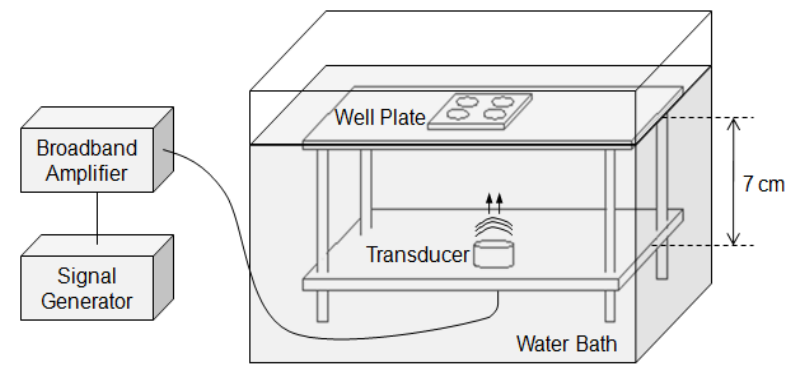

Fig. 1. Overview of exposure apparatus. The probe and the acousticallytransparent well plate was separated by $7 \mathrm{~cm}$ apart. During operation, pulses from the signal generator were amplified before being sent through the probe.

growing in the asynchronous exponential phase were then harvested with a $10^{6}$ cells/ml concentration as estimated using a hemocytometer (Marienfeld Laboratory Glassware). $1 \mathrm{ml}$ of the harvested cells was subsequently transferred to one of the wells in an acoustically transparent four-well plate (176740; Nunc) for each run of the experiment.

\section{B. Microbubble Preparation}

To facilitate sonoporation during the ultrasound exposure, a $10 \mu \mathrm{l}$ microbubble solution ( $1 \%$ volume concentration) was added to the $1 \mathrm{ml}$ cell suspension extract used for each exposure trial. The microbubbles used in this work were made via sonication from perfluorobutane gas, and their shell consisted of a lipid monolayer formed via phosphatidylcholine and polyethylene glycol stearate (courtesy of Prof. Alexander Klibanov, University of Virginia). The original density of these microbubbles was roughly $10^{6}$ bubbles $/ \mathrm{ml}$, and their size ranged from 1-5 $\mu \mathrm{m}$ [12].

\section{Ultrasound Exposure Protocol}

In each experiment run, the well plate containing the cellmicrobubble mixture sample was placed at the top of the ultrasound exposure apparatus as shown in Fig. 1. The well plate, sealed with a parafilm layer to avoid possible contamination, was put in contact with the apparatus's water bath surface that served as the acoustic coupling medium. Ultrasound pulses were then insonated for $1 \mathrm{~min}$. at the well plate using a $1 \mathrm{MHz}$ unfocused ultrasound probe with $2.54 \mathrm{~cm}$ diameter (P1T10W-25; Kunshan Risun Electronic Co., Jiangsu, China) that was positioned at $7 \mathrm{~cm}$ away from the well plate. The transmitted ultrasound pulses had a 10\% duty-cycle and a $1 \mathrm{kHz}$ pulse repetition frequency as shaped using an arbitrary signal generator (33120A; Agilent Technologies). Also, they were amplified using a broadband power amplifier (ENI 2100L; Electronics \& Innovation Ltd) to reach a 0.5 MPa peak negative pressure at $7 \mathrm{~cm}$ away from the probe, which was determined by a membrane hydrophone (HMB-0500; Onda Corporation) in the absence of the acoustically-transparent well plate. This ultrasound pressure level, in the presence of microbubbles, is known to be sufficient to induce acoustic cavitation without significant thermal elevation [8]. It should be noted that standing waves may be present within the cellmicrobubble mixture since the top of the well plate was coupled to air, and thus the amount of sonoporation activity may be increased [4].

\section{Scanning Electron Microscopy Analysis}

To verify that sonoporation was induced in the experiments, scanning electron microscopy (SEM) was performed on the cell samples of a few pilot experiment runs during and after ultrasound exposure. For these pilot trials, the ultrasoundexposed cell samples were first fixed with $2.5 \%$ glutaraldehyde solution either a few seconds into the ultrasound exposure or 1 min. after the end of exposure. The fixed cells were stored at $4^{\circ} \mathrm{C}$ for over $4 \mathrm{~h}$; after that they were rinsed with phosphate buffered saline and were dehydrated repeatedly using ethanol with increasing concentration (30\%, 50\%, 70\%, 90\%, 100\%) to reduce the cells' surface tension as necessary for SEM analysis. After the dehydrated cells were dried, they were analyzed under a field emission SEM with a $5 \mathrm{kV}$ operating voltage (S4800 FEG; Hitachi). SEM photos of the cell membrane morphology at the two fixation times were taken as direct observations of sonoporation.

\section{E. Western Blot Analysis}

To investigate the cytoplasmic signaling changes involved as a result of sonoporation, western blot analysis of signaling proteins was carried out on the cell samples after the ultrasound exposure. In preparing for this analysis, the ultrasound-exposed cells were first re-incubated at $37^{\circ} \mathrm{C}$ in a $5 \% \mathrm{CO}_{2}$ air atmosphere. They were subsequently harvested at four time points ( $4 \mathrm{~h}, 8 \mathrm{~h}, 12 \mathrm{~h}, 24 \mathrm{~h}$ ), and approximately $5 \times 10^{6}$ cells were collected from each sample. The protein contents in each sample were then separated according to molecular weight using sodium-dodecyl-sulfate polyacrylamide gel electrophoresis (EPS 301; Amersham Pharmacia Biotech), and the results were transferred onto polyvinylidene fluoride membranes using a semidry blotter (TE 77 PWR; GE Life Sciences). After that, antibody staining was applied to the separated protein contents on the blot membranes to examine the expression level of major signaling proteins at the four time points. In particular, five signaling proteins related to apoptosis and mitosis were stained in this analysis:

- $\underline{\text { PARP }}$ (poly-ADP-ribose polymerase): A major DNA repair protein that acts against apoptosis (ab6079; Abcam)

- Cleaved PARP: Inactivated PARP proteins due to cleavage by pro-apoptotic caspase-3 proteins (ab6079; Abcam)

- Bcl-2 (B-cell lymphoma 2): Inhibitor for mitochondrial release of pro-apoptotic molecules (sc-7382; Santa Cruz)

- Bax (Bcl-2-associated X): Promoter for pro-apoptotic releases from mitochondria (sc-6236; Santa Cruz)

- Cdc-2 (cell division control protein 2): Checkpoint for entering mitotic phase of cell cycle (ab32384; Abcam)

The final staining results were transferred to X-ray films and were converted into grayscale images for display. Note that the staining was allowed to take place over two hours to facilitate contrast development, after which secondary staining was performed for one hour using goat anti-mouse and anti-rabbit antibodies (170-6515 \& 170-6516; Bio-Rad) to enhance the contrast. Also, to prevent non-specific background staining, the blot membranes were immersed in non-fat milk before the staining commenced. 
A

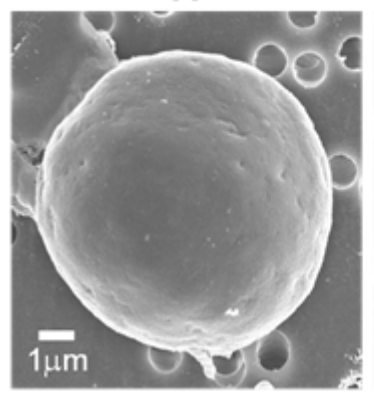

B

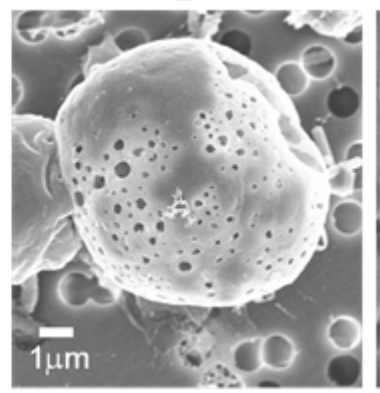

B (Zoomed)

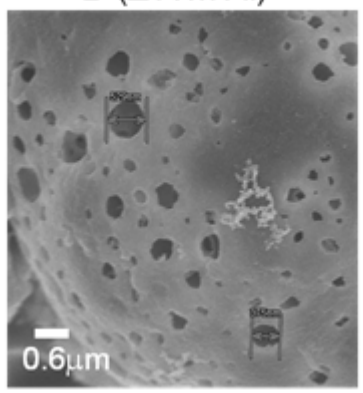

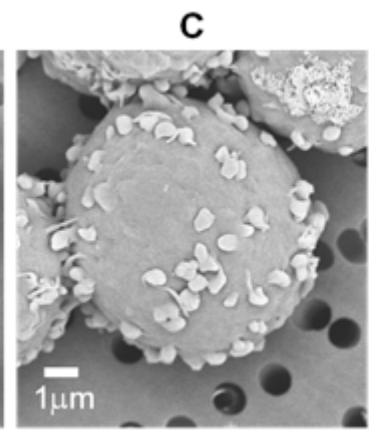

C (Zoomed)

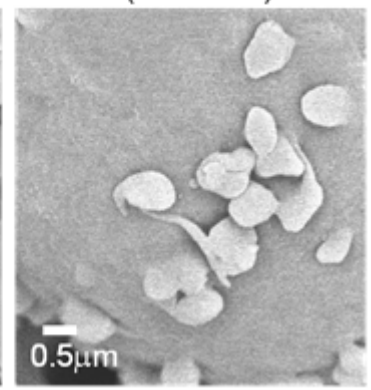

Fig. 2. SEM images of HL-60 leukemia cells that received: (a) sham exposure; (b) ultrasound exposure for a few seconds (1 MHz center frequency, $10 \%$ duty cycle, $1 \mathrm{kHz}$ pulse repetition frequency, $0.5 \mathrm{MPa}$ peak negative pressure, $1 \%$ microbubbles); (c) 1 min. ultrasound exposure (same parameters as (b)) followed by 1 min. recovery time.
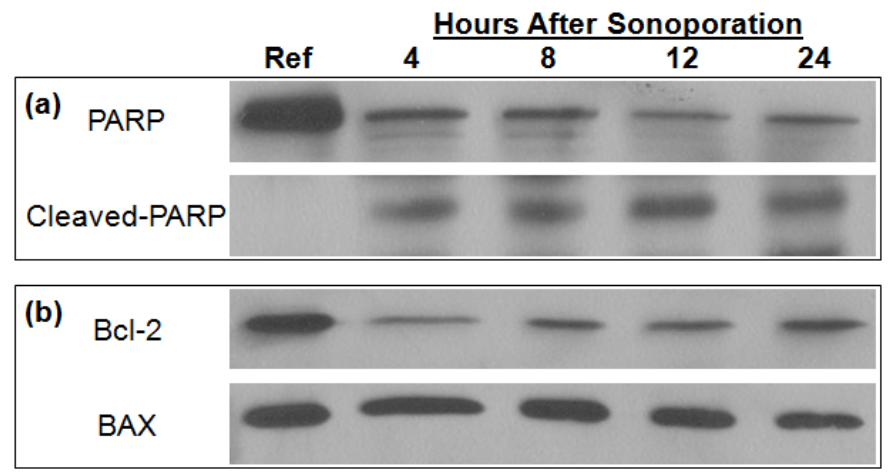

(c)

Cdc-2

Fig. 3. Western blot of cytoplasmic signaling proteins at $4 \mathrm{~h}, 8 \mathrm{~h}, 12 \mathrm{~h}$, and $24 \mathrm{~h}$ after sonoporation. Darker and thicker bands indicate stronger protein expression levels. Results for these proteins are shown: (a) apoptosis indicators (PARP, cleaved-PARP); (b) mitochondrial regulators related to apoptosis (Bcl-2, Bax); (c) mitosis checkpoint (Cdc-2).

\section{RESULTS}

\section{A. Direct Observations of Sonoporation}

Fig. 2 shows a series of SEM photos on representative HL60 leukemia cells that received different ultrasound exposures. It can be seen that for the sham exposure case (Fig. 2a), the cell membrane remained intact and looked spherical in shape. In contrast, for the case with few-second ultrasound exposure (Fig. 2b), the SEM photos showed that some cells had randomly-positioned pores appearing on the membrane. This represents direct observations that membrane poration had took place as a result of exposing the cell-microbubble mixture to the pulsed ultrasound level used in our experiment. It is worth noting that the majority of these holes were found to have a diameter between 0.1-0.5 $\mu \mathrm{m}$.

For the case taken $1 \mathrm{~min}$. after the end of ultrasound exposure (Fig. 2c), two observations of interest should be noted. First, an intact membrane can be found on many of the cells, indicating that most cells had resealed their membrane within minutes after they had experienced sonoporation. Second, the membrane surface of these resealed cells had a number of scattered patches that were not seen in the sham-exposure cells in Fig. 2a, and the size of these patches seemed to be on the same order as the membrane pores seen in Fig. 2b. This may imply that the resealing process following sonoporation may involve a patching mechanism.

\section{B. Activation of Apoptotis Signaling After Sonoporation}

Fig. 3a shows the expression levels of apoptosis indicator proteins studied in this work (PARP, cleaved-PARP). Regular PARP levels and no cleaved-PARP expression can be observed for the sham exposure case (left column). This should be compared with the results for ultrasound-exposed cells $4-24 \mathrm{~h}$ after experiencing sonoporation (four right columns), where the PARP level was dampened whilst cleaved-PARP expression became apparent. Such an observation suggests that sonoporation may lead to cellular apoptosis by activating the signaling pathway that involves PARP cleavage. Note that the apoptotic signaling appeared to be the strongest after $12 \mathrm{~h}$.

\section{Role of Mitrochondrion in Apoptotis Signaling After Sonoporation}

Fig. 3b shows the expression levels of Bcl-2 and Bax, which are two major regulator proteins found on the membrane of the mitochondrion (an organelle with a gate-keeping role in apoptosis). For the sham exposure case (left column), it can be seen that the levels of Bcl-2 (pro-survival) and Bax (proapoptosis) were maintained in balance. In contrast, for the sonoporation-treated cells (four right columns), Bcl-2 had dropped whilst Bax had increased (most substantial after $4 \mathrm{~h}$ ). These conditions favored increases in mitochondrial secretion of signaling molecules that activate apoptosis. Note that the ratio imbalance between Bcl-2 and Bax became less significant over time, and thus apoptotic signaling from mitochondria seemed to be gradually dampened.

\section{Repression of Mitosis Signaling After Sonoporation}

Fig. 3c shows the expression level of the Cdc-2 protein that is responsible for initiating the mitotic phase (M-phase) of the cell cycle. As can be seen, Cdc-2 had reduced in expression following sonoporation (lowest after $4 \mathrm{~h}$ ). This is an indication that cell division was temporarily down-regulated after sonoporation was experienced (likely to facilitate cellular repair). Such repression seemed to be gradually revoked over time. 


\section{DISCUSSION}

Based on the findings of this study, one inference that can be drawn is that sonoporated cells may respond to their disrupted intracellular equilibrium by up-regulating cytoplasmic signals related to apoptosis and cell-cycle arrest. Such a response can be regarded as an indication that some of the sonoporated cells have been irreversibly injured despite maintaining short-term viability. For these cells, mitosis is halted through down-regulation of the Cdc-2 checkpoint protein (Fig. 3c) that is crucial for cells to enter the M-phase of the cell cycle [13]. Concurrently, apoptosis may be triggered in these cells through their mitochondrion, where a drop in Bcl-2 and a rise in Bax (Fig. 3b) are known to permeabilize the mitochondrial membrane and thereby promote the efflux of a pro-apoptotic molecule known as cytochrome-c into the cytoplasm [14]. The pathway leading to the apoptosis of these cells likely involves PARP cleavage (Fig. 3a), an event that is known to be caused by the activation of the caspase-3 protein (a common pathway for apoptosis execution) [15].

Perhaps one question that warrants further investigation is how the mitochondrion detects cellular trauma attributed to sonoporation and thereby upregulates its apoptotic signaling activities. We hypothesize that the intracellular calcium ion concentration may play an important role here, given that elevations of this ion is known to take place after sonoporation [11] and it is recognized as a pro-apoptotic stimulus for the mitochondrion [14]. Confirmation of this relationship can help better reveal the cytoplasmic mechanism for sonoporationinduced apoptosis and in turn design better cellular repair strategies for sonoporation-based drug delivery paradigms.

Another question of interest is how the membrane reseals after cells experience sonoporation. The scattered patches seen on the resealed cell membrane (Fig. 2c) give indication that the resealing process may be more sophisticated than a simple self-reunion of the membrane. Instead, we hypothesize that resealing may follow an exocytotic patching mechanism whereby cytoplasmic vesicles are first delivered to the poration site and are then fused together with the membrane to fill up the hole [16]. Such a mechanism is known to take place when there is a calcium ion influx into porous cells, as is the case for sonoporated cells [11].

\section{CONCLUSION}

This work has been set out to gain insights on the cytoplasmic signaling changes involved in sonoporationinduced apoptosis and cell-cycle arrest. It was found that sonoporation may induce apoptosis through the mitochondrion and follows a signaling pathway involving pro-apoptotic caspase proteins. Also, sonoporated cells may slow down their mitosis through down-regulating cell-cycle checkpoint proteins. Based on these findings, it seems that unfavorable risk on longterm cell viability is involved in using sonoporation for drug delivery purposes, and it may be necessary to develop cellular repair strategies for such applications. On the other hand, our findings may represent new opportunities in using sonoporation for other therapeutic purposes. For instance, with the design of targeted microbubbles, it may be possible to use sonoporation as a site-specific mechanism for suppressing cancer cell growth.

\section{ACKNOWLEDGMENTS}

We are grateful to Prof. Alexander Klibanov (University of Virginia) for preparing the microbubbles used in this work. We also thank Prof. Chien Ting Chin (Shenzhen University), Dr. Jade Shi (Hong Kong Baptist University), and Dr. Barbara Chan (University of Hong Kong) for their valuable advices.

\section{REFERENCES}

[1] C. X. Deng, F. Sieling, H. Pan, and J. Cui, "Ultrasound-induced cell membrane porosity”, Ultrasound Med. Biol., vol. 30, pp. 519-526, 2004.

[2] S.Mehier-Humbert, T. Bettinger, F.Yan, and R. H. Guy, "Plasma membrane poration induced by ultrasound exposure: implication for drug delivery”, J. Control. Release, vol. 104, pp. 213-222, 2005.

[3] A. van Wamel, K. Kooiman, M. Harteveld, and M. Emmer, "Vibrating microbubbles poking individual cells: Drug transfer into cells via sonoporation”, , J. Control. Release, vol. 112, pp. 149-155, 2006.

[4] H. D. Liang, J. Tang, and M. Halliwell, “Sonoporation, drug delivery, and gene therapy”, Proc. Inst. Mech. Eng. H, vol. 224, pp. 343-361, 2010.

[5] H. R. Guzman, D. X. Nguyen, S. Khan, and M. R. Prausnitz, "Ultrasound-mediated disruption of cell membranes. I. Quantification of molecular uptake and cell viability”, J. Acoust. Soc. Am., vol. 110, pp. 588-596, 2001.

[6] T. A. Tran, S. Roger, J. Y. Le Guennec, F. Tranquart, and A. Bouakaz, "Effect of ultrasound-activated microbubbles on the cell electrophysiological properties”, Ultrasound Med. Biol., vol. 33, pp. 158-163, 2007.

[7] L. J. M. Juffermans, P. A. Dijkmans, R. J. P. Musters, C. A. Visser, and O. Kamp, "Transient permeabilization of cell membranes by ultrasoundexposed microbubbles is related to formation of hydrogen peroxide”, Am. J. Physiol. Heart Circ. Physiol., vol. 291, pp. H1595-H1601, 2006.

[8] R. Karshafian, P. D. Bevan, R. Willianms, S. Samac, and P. N. Burns, "Sonoporation by ultrasound-activated microbubble contrast agents: effect of acoustic exposure parameters on cell membrane permeability and cell viability,” Ultrasound Med. Biol., vol. 35, pp. 847-860, 2009.

[9] H. Ando, H. L. B. Feril Jr, T. Kondo, Y. Tabuchi, R. Ogawa, Q. L. Zhao, Z. G. Cui, S. Umemura, H. Yoshikawa, and T. Misaki, "An echocontrast agent, Levovist, lowers the ultrasound intensity required to induce apoptosis of human leukemia cells,” Cancer. Lett., vol. 242, pp. 37-45, 2006.

[10] D. L. Miller and C. Dou, "Induction of apoptosis in sonoporation and ultrasonic gene transfer”, Ultrasound Med. Biol., vol. 35, pp. 144-154, 2009.

[11] J. D. Hutcheson, R. K. Schlicher, H. K. Hicks, and M. R. Prausnitz, "Saving cells from ultrasound-induced apoptosis: quantification of cell death and uptake following sonication and effects of targeted calcium chelation”, Ultrasound Med. Biol., vol. 36, pp. 1008-1021, 2010.

[12] C. T. Chin, B. I. Raju, T. Shevchenko, and A. L. Klibanov, "Control and reversal of tumor growth by ultrasound activated microbubbles", Proc. IEEE Ultrason. Symp., pp. 77-80, 2009.

[13] J. A. Pietenpol and Z. A. Stewart, "Cell cycle checkpoint signaling: cell cycle arrest versus apoptosis”, Toxicology, vol. 181, pp. 475-481, 2002.

[14] Y. Rong and C. W. Distelhorst, "Bcl-2 protein family members: versatile regulators of calcium signaling in cell survival and apoptosis”, Annu. Rev. Physiol., vol. 70, pp. 73-91, 2008.

[15] R. C. Taylor, S. P. Cullen, and S. J. Martin, “Apoptosis: controlled demolition at the cellular level”, Nat. Rev. Mol. Cell Biol., vol. 9, pp. 231-241, 2008.

[16] P. L. McNeil, "Plasma membrane disruption: repair, prevention, adaptation,” Annu. Rev. Cell Dev. Biol., vol. 19, pp. 697-731, 2003. 